\title{
GNE myopathy
}

INSERM

\section{Source}

INSERM. (1999). Orphanet: an online rare disease and orphan drug data base. GNE myopathy. ORPHA:602

GNE myopathy is a rare autosomal recessive distal myopathy characterized by early adult-onset, slowly to moderately progressive distal muscle weakness that preferentially affects the tibialis anterior muscle and that usually spares the quadriceps femoris. Muscle biopsy reveals presence of rimmed vacuoles. 